\title{
ROLE OF HRCT IN EVALUATION OF BRONCHIECTASIS
}

\author{
M. Vijaya Kumari1 , J. S. Aswini Jyothi², K. Sapna ${ }^{3}$
}

${ }^{1}$ Associate Professor, Department of Radiology, Osmania Medical College/Hospital, Hyderabad.

${ }^{2}$ Assistant Professor, Department of Radiology, Osmania Medical College/Hospital, Hyderabad.

3Postgraduate, Department of Radiology, Kakatiya Medical College/MGM General Hospital, Warangal.

ABSTRACT
BACKGROUND
Bronchiectasis is defined as a localised, irreversible dilatation of the bronchial tree. Bronchiectasis is a chronic and progressive
lung disease and can lead to chronic respiratory failure. HRCT has emerged as the imaging modality of choice for evaluating
suspected cases of bronchiectasis. HRCT is excellent in characterisation of the type of bronchiectasis and extent of lung involvement.

AIMS

1. To study the role of HRCT in diagnosis of bronchiectasis.

2. To study regional distribution of bronchiectasis on HRCT.

3. To characterise bronchiectasis morphologically and to determine aetiologies of bronchiectasis wherever possible.

\section{MATERIAL AND METHODS}

A prospective study of 50 patients was done in the Department of Radiology and Imaging at Kakatiya Medical College and MGM General Hospital, Warangal.

\section{PATIENT SELECTION}

Patients of all age groups were selected in whom clinically bronchiectasis was suspected. Ours was a random study for a period of three years and no specific criteria were laid down for patient selection. From May 2005 - April 2008, 50 patients clinically suspected to have bronchiectasis were subjected to chest radiograph and HRCT examination.

\section{RESULTS}

In our study by co-relating HRCT findings and clinical history an accurate diagnosis was made in 46 cases, i.e. $76.6 \%$ which was confirmed by laboratory investigations. In our study 23 patients (46\%) had pulmonary tuberculosis, in 8 patients (16\%) no cause was found, 16 patients (32\%) had history of recurrent childhood infections, 2 patients (4\%) had bronchial asthma and COPD and 1 patient $(2 \%)$ had allergic bronchopulmonary aspergillosis. In this study incidence was common in the age group of 21-30 years and is more common in males than females. Bilateral involvement is more common than unilateral involvement.

\section{CONCLUSION}

In a given clinical setting suggestive of bronchiectasis, HRCT serves as the best modality in confirming the diagnosis of bronchiectasis. HRCT is definitely superior to chest radiographs in the detection of bronchiectasis.

\section{KEYWORDS}

HRCT bronchiectasis, Cylindrical, Cystic, Varicose Bronchiectasis.

HOW TO CITE THIS ARTICLE: Kumari MV, Jyothi JSA, Sapna K. Role of HRCT in evaluation of bronchiectasis. J. Evolution Med. Dent. Sci. 2016;5(77):5728-5737, DOI: 10.14260/jemds/2016/1292

\section{INTRODUCTION}

Bronchiectasis is defined as a localised, irreversible dilatation of the bronchial tree. Although, a wide variety of disorders have been associated with bronchiectasis, it most commonly results from acute, chronic or recurrent infections and cystic fibrosis. Bronchiectasis is commonly acquired during childhood, the condition may rarely result from a gross

Financial or Other, Competing Interest: None.

Submission 18-07-2016, Peer Review 05-08-2016,

Acceptance 11-08-2016, Published 26-09-2016.

Corresponding Author:

Dr. J. S. Aswini Jyothi,

Flat: 204, Vasavi Bhuvana Apartments,

H: 8-3-981/1, 3,4,6,8,10,11

Srinagar Colony, Hyderabad-500037,

Telangana.

E-mail: jyothijs@yahoo.com

DOI: $10.14260 /$ jemds/2016/1292 congenital developmental anomaly or may be predisposed by some other inherited defect either ultra-structural (e.g. ciliary dyskinesia which includes Kartagener's and Young's syndrome) or related to a generalised metabolic defect (e.g. Cystic fibrosis and Alpha- 1 antitrypsin deficiency) or due to an immunodeficiency syndrome (e.g. hypogammaglobulinaemia). Post infective is the most common cause and may result from measles, necrotising bacterial pneumonias, granulomatous disease, allergic bronchopulmonary aspergillosis or from bronchial obstruction as in cases of inhaled foreign body.

The presentation includes recurrent respiratory tract infections, productive cough, dyspnoea and occasionally haemoptysis.

\section{Treatment}

Is with antibiotics and surgical resection is indicated. Surgery in patients with bronchiectasis is indicated when medical treatment fails if there is obstructing tumour or foreign body, 
if life-threatening complications occur such as uncontrolled haemorrhage.

\section{Imaging}

With conventional radiography, the accuracy is $65 \%$ to $80 \%$. Documentation of the disease has traditionally relied on bronchography, which is rarely performed now.

HRCT has largely eliminated the need for bronchography in the diagnosis of bronchiectasis. HRCT is currently the most sensitive tool for non-invasive imaging of the lung parenchyma. It allows acquisition of in vivo images with spatial resolution comparable to direct visualisation of the lung slices. HRCT techniques have evolved over the last 10 years together with advance in CT technology. These have resulted in increased spatial resolution and decreased scan time leading to marked improvements in image quality. The unique high contrast between lung parenchyma and air provides an ideal situation for HRCT scanning. Although, its use was initially controversial, volumetric CT with thin sections is superior to conventional high-resolution CT technique in the detection of bronchiectasis. According to recent study, volumetric CT with thin sections is superior to conventional high-resolution CT technique in the detection of bronchiectasis.

However, when using the same exposure factors MDCT results in considerably greater radiation dose (August 2006 edition of the American Journal of Roentgenology). The first use of the term high resolution CT (HRCT) has been attributed to Todo et al who in 1982, described the potential use of this technique for assessing lung disease. The first reports of HRCT in English, date to 1985, including landmark descriptions of HRCT findings by Nakata, Naidich and Zerhouni. The HRCT technique has resulted in: 1) Increased spatial resolution, 2) Decreased scan time leading to, 3) Marked improvement in image quality. The unique high contrast between lung parenchyma and air provides an ideal situation for HRCT scanning.

\section{MATERIALS AND METHODS}

A prospective study of 50 patients was done in the Department of Radiology and Imaging at Kakatiya Medical College and MGM General Hospital, Warangal.

\section{Patient Selection}

Patients of all age groups were selected in whom clinically bronchiectasis was suspected. Ours was a random study and no specific criteria were laid down for patient selection. From May 2005 - April 2008, 50 patients clinically suspected to have bronchiectasis were subjected to chest radiograph and HRCT examination.

\section{Technical Consideration}

All HRCT scans were performed at our hospital on the Toshiba Asteion CT Scanner. The patients were placed supine and no gantry tilt was given. Scout films were taken routinely in all patients before starting the scan. Scanning commenced from lung apices to lung bases. Scans were performed in suspended inspiration. Lung window setting was used with window width of 1500 to $1600 \mathrm{HU}$ and window level of -600 to -700 HU. HRCT was performed obtaining $1 \mathrm{~mm}$ intervals. A high algorithm was used with kvp of 120 and mA of 150 .

\section{OBSERVATIONS AND RESULTS}

We did a random study in 50 patients in our institute to evaluate the HRCT findings of bronchiectasis. All these patients were clinically suspected to be suffering from this disease.

Out of a total of 50 patients there were $29(58 \%)$ males and 21 (41\%) females, the average age groups being 21-30 years.

\begin{tabular}{|c|c|c|c|}
\hline Age in Years & Male & Female & Total \\
\hline $0-10$ & - & 2 & 2 \\
\hline $11-20$ & 2 & 2 & 4 \\
\hline $21-30$ & 12 & 8 & 20 \\
\hline $31-40$ & 10 & 2 & 12 \\
\hline $41-50$ & 2 & 5 & 7 \\
\hline $51-60$ & 1 & 2 & 3 \\
\hline $61-70$ & 2 & 0 & 2 \\
\hline \multicolumn{3}{|c|}{ Table 1: Age \& Sex Incidence } \\
\hline
\end{tabular}

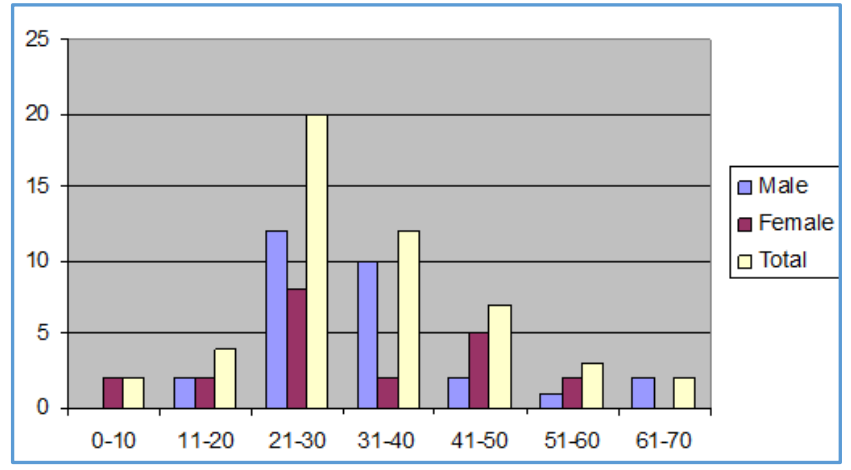

We thus concluded that the maximum number of patients $(n=20)$ were in the age group of $21-30$ years $(40 \%)$. There were only 2 patients below the age of 10 and above $60 \mathrm{yrs}$.

Majority of male and female patients in our study were in the age group of 21-30 years.

\begin{tabular}{|c|c|c|}
\hline & No. of Patients & $\%$ \\
\hline Tuberculosis & 23 & 46 \\
\hline Recurrent Infections & 16 & 32 \\
\hline Aspergillosis & 1 & 2 \\
\hline Bronchial Asthma & 2 & 4 \\
\hline Idiopathic & 8 & 16 \\
\hline \multicolumn{3}{|c|}{ Table 2: Possible Aetiological Factors in our Study } \\
\hline
\end{tabular}

Tuberculosis was identified as the cause of bronchiectasis in 23 patients ( $46 \%$ ) and is the major case of bronchiectasis in our study.

\section{The Lobar Distribution was as Follows}

15 patients (30\%) had unilateral disease and 35 (70\%) had disease in both lungs.

\begin{tabular}{|c|c|c|c|c|}
\hline & Right & Left & No. of Patients & \% \\
\hline Unilateral & 2 & 13 & 15 & $30 \%$ \\
\hline Bilateral & & & 35 & $70 \%$ \\
\hline \multicolumn{5}{|c|}{ Table 3: Localisation of Bronchiectasis } \\
\hline
\end{tabular}

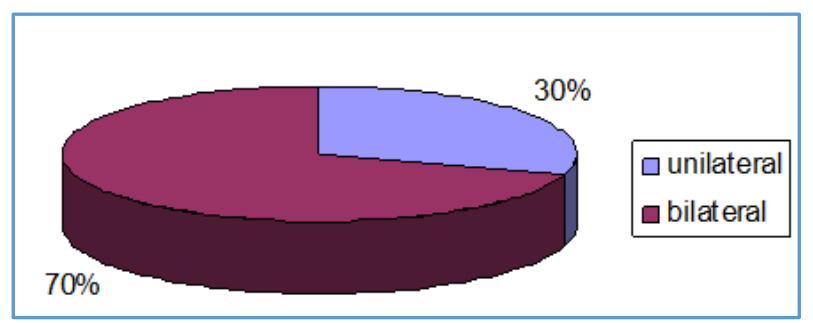




\begin{tabular}{|c|c|c|c|}
\hline Location & Right & Left & Total \\
\hline Upper Lobe & 26 & 21 & 47 \\
\hline Middle (Lingual) & 29 & 25 & 54 \\
\hline Lower lobe & 27 & 41 & 68 \\
\hline Total & $\mathbf{8 2}$ & $\mathbf{8 7}$ & $\mathbf{1 6 9}$ \\
\hline Table 4: Regional (Lobar) Distribution of Bronchiectasis \\
\hline
\end{tabular}

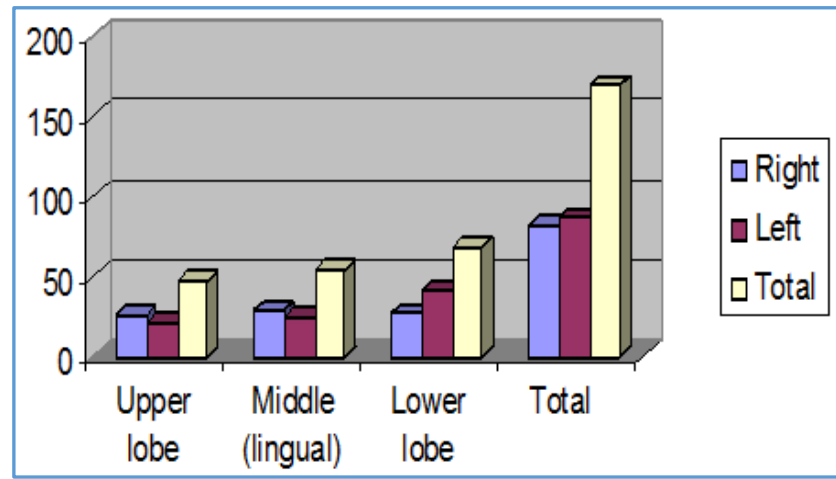

There was thus a predominance of lower lobe affliction $(40.2 \%)$ in our study.

\begin{tabular}{|c|c|c|c|c|}
\hline Site & Lobe & Segment & Number & Percent \\
\hline \multirow[t]{10}{*}{ Right } & Upper & Apical & 9 & 18 \\
\hline & & Anterior & 25 & 50 \\
\hline & & Posterior & 6 & 12 \\
\hline & Middle & Medial & 28 & 56 \\
\hline & & Lateral & 17 & 34 \\
\hline & Lower & Superior & 12 & 24 \\
\hline & & Medial Basal & 13 & 26 \\
\hline & & Posterior Basal & 12 & 24 \\
\hline & & Lateral Basal & 5 & 10 \\
\hline & & Anterior Basal & 16 & 32 \\
\hline \multirow[t]{8}{*}{ Left } & Upper & Apicoposterior & 7 & 14 \\
\hline & & Anterior & 17 & 34 \\
\hline & Lingual & Superior & 25 & 50 \\
\hline & & Inferior & 20 & 40 \\
\hline & Lower & Superior & 25 & 50 \\
\hline & & Posterior Basal & 36 & 72 \\
\hline & & Lateral Basal & 17 & 34 \\
\hline & & Anterior Basal & 20 & 40 \\
\hline & Tabl & 5: Segmental D & ibution & \\
\hline
\end{tabular}

Posterior basal segment of left lower lobe was most commonly affected $(72 \%)$ followed by medial segment of right middle lobe (56\%).

\begin{tabular}{|c|c|c|c|}
\hline No. & Morphological Forms & No. of Cases & \% \\
\hline 1. & Cystic & 24 & 48 \\
\hline 2. & Cylindrical & 5 & 10 \\
\hline 3. & Varicose & 2 & 4 \\
\hline 4. & Mixed & 19 & 38 \\
\hline \multicolumn{3}{|c|}{ Table 6: Morphological Forms of Bronchiectasis } \\
\hline
\end{tabular}

Cystic bronchiectasis was the most common (48\%) morphological form of bronchiectasis followed by mixed bronchiectasis.

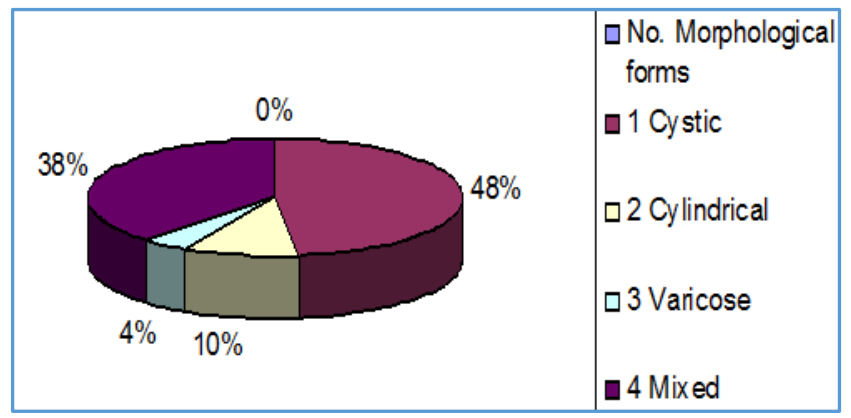

\begin{tabular}{|c|c|c|}
\hline HRCT & $\begin{array}{c}\text { No. of } \\
\text { Patients }\end{array}$ & $\mathbf{\%}$ \\
\hline Lack of tapering & 38 & 76 \\
\hline $\begin{array}{c}\text { Bronchial : Pulmonary artery ratio > } \\
\text { 1.5 }\end{array}$ & 31 & 62 \\
\hline Bronchial wall thickening & 37 & 74 \\
\hline Peripherally visualised bronchi & 40 & 80 \\
\hline $\begin{array}{c}\text { Air fluid level/Dilated mucous filled } \\
\text { bronchi }\end{array}$ & 20 & 40 \\
\hline \multicolumn{2}{|c|}{ Table 7: HRCT Findings } \\
\hline \multicolumn{2}{|c}{}
\end{tabular}

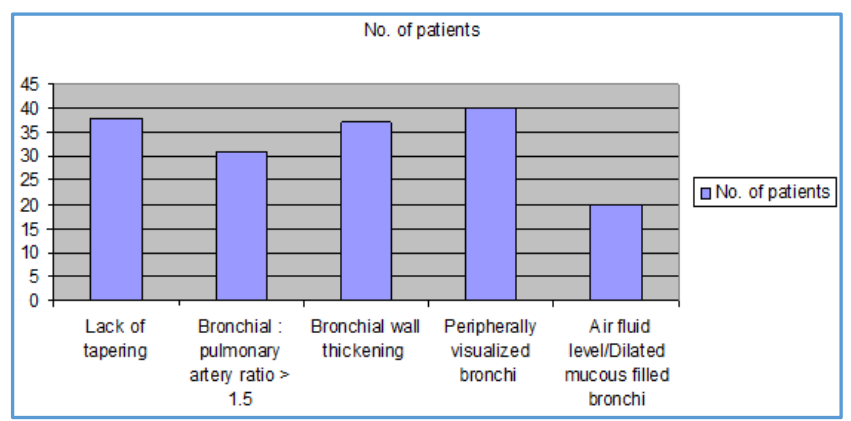

Peripherally visualised bronchi (80\%) was the most common findings in our study followed by lack of tapering (76\%).

Bronchial wall thickening was seen in $74 \%$ of patients. Bronchial : Pulmonary artery ratio was noted in $62 \%$ of patients.

\begin{tabular}{|c|c|c|}
\hline & Radiograph & HRCT \\
\hline True +ve & 48 & 50 \\
\hline False -ve & 2 & - \\
\hline False +ve & - & - \\
\hline \multicolumn{2}{|c|}{ Table 8: Radiograph V/S HRCT } \\
\hline
\end{tabular}

\begin{tabular}{|c|c|}
\hline Associated Findings & No. of Patients \% \\
\hline Emphysema & 11 \\
\hline Consolidation & 16 \\
\hline Collapse & 17 \\
\hline Pleural thickening & 8 \\
\hline Cavitation & 15 \\
\hline Fibrosis & 10 \\
\hline Dextrocardia & 2 \\
\hline Aspergilloma & 1 \\
\hline Table 9: Associated Findings Along with Bronchiectasis \\
\hline
\end{tabular}

Many patients has more than one of the above findings. Emphysema was noted in 11 patients, collapse in 17 patients, consolidation in 16 patients. 


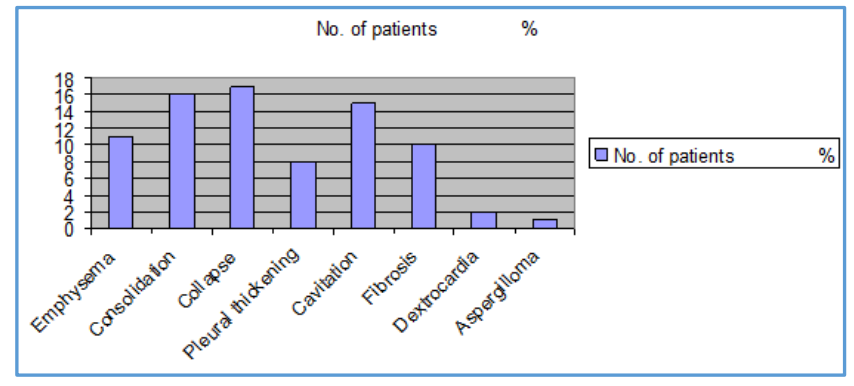

\section{DISCUSSION}

Bronchiectasis has been defined as localised, irreversible abnormal dilatation of the bronchial tree. Although, a wide variety of disorders have been associated with it, bronchiectasis most commonly occurs from acute, chronic or recurrent infections.

In general a clinical diagnosis of bronchiectasis is possible only in the most severely affected patients and even in this setting differentiation from chronic bronchitis may be difficult. Most patients present with purulent sputum production and recurrent pulmonary infections. Haemoptysis is also frequent and may be the only clinical finding.

Although, traditionally considered the "gold standard," the reliability of bronchography in the diagnosis of bronchiectasis has been called into question. Currie et al (1987) in a study of 27 patients with chronic sputum production evaluated bronchographically showed that there was a significant interobserver variability when studies were interpreted by two well-trained bronchographers. Agreement was reached only in 19 out of 27 (70\%) by one radiologist only. These findings suggest that bronchography may be more limited in its utility than previously thought. ${ }^{1}$

We did a random study in 50 patients in our hospital who were clinically suspected to have bronchiectasis. HRCT's were performed on these patients on Toshiba sub-second Spiral CT Scan.

Scans were performed by obtaining $1 \mathrm{~mm}$ sections at 10 $\mathrm{mm}$ interval from the thoracic inlet to diaphragm using a high algorithm. This was in accordance with Webb et al who suggested that in patients in whom there are no specific clinical or radiological signs to help localise the disease, $1 \mathrm{~mm}$ or $1.5 \mathrm{~mm}$ high resolution images should be obtained every 10 $\mathrm{mm}$ from the lung apices to bases. Despite the lack of contiguous scanning, this technique allows adequate assessment of the interstitium in nearly all cases.

This approach can be modified to reflect various clinical presentations. For example, in patients presenting with haemoptysis it is usually necessary to rule out occult central endobronchial lesions in addition to detecting bronchiectasis. This is accomplished by obtaining $1 \mathrm{~mm}$ to $1.5 \mathrm{~mm}$ thick sections every $10 \mathrm{~mm}$ through the upper and lower lung zones and contiguous $5 \mathrm{~mm}$ thick sections from carina to level of inferior pulmonary vein.

A high algorithm and a scan time of $1-2$ seconds and 120 KV tube voltage and MA of 150 were used in our study.

While performing the HRCT's, we used lung window settings with a window width of 1500-1700 HU and Window level of - 600 --- $700 \mathrm{HU}$.

\section{The Data of the Present Studies are Discussed as follows} Age Incidence

In the present series, age of patients varied between as young as 9 yrs. to as old as 70 yrs. The maximum number of cases (20) $40 \%$ were in the age group of $21-30$ yrs.

\section{Year Age Group}

Present series 2007, 21 - 30 yrs.

Fazul Gela et al 1999, 31 - 40 yrs.

Davies, et al 1991, 21 - 30 yrs.

The present study matches with the study of Davies et al. It has been seen that with advancing years, the incidence is increasing in the younger individuals. This could be due to increasing pollution and also because of early presentation of patients because of increased awareness and early detection due to better imaging modalities.

\section{Sex Incidence}

In the present series, there were 21 (42\%) females and 29 (58\%) males.

\begin{tabular}{|c|c|c|c|}
\hline & Year & Male & Female \\
\hline Present Series & 2007 & 29 & 21 \\
\hline Pasteur MC et al & 2000 & 56 & 94 \\
\hline Fazul Gelal et al & 1999 & 5 & 11 \\
\hline Zaleska M et al & 1999 & 24 & 45 \\
\hline
\end{tabular}

\section{Symptoms}

Cough was the major symptom and was seen in all 60 (100\%) cases. Most of the patients except 5 had productive cough.

In the study done by Frey HR and Russi et al (1997), ${ }^{2}$ daily sputum production was the most common symptom. In a clinical study done by Guleria in 1996, 92\% of patients had cough and sputum as presenting symptoms. Total daily sputum production has been used to characterise severity of bronchiectasis. According to Issac Hassan et al (2003) production of less than $10 \mathrm{~mL} /$ day is defined as mild bronchiectasis, $10-15 \mathrm{~mL} /$ day is defined as moderate bronchiectasis and more than $15 \mathrm{~mL} /$ day is defined as severe bronchiectasis.

In studies conducted by Bindra (1987) and Guleria (1996) haemoptysis was noted in 58 and $60 \%$, respectively. In our study, $41.6 \%$ of patients presented with haemoptysis. Haemoptysis occurs in $50-70 \%$ of cases and can be due to bleeding from friable, inflamed airway mucosa. More significant massive bleeding is often a consequence of bleeding from hypertrophied bronchial arteries. These hypertrophied bronchial arteries can be detected as nodular and tubular structures in the mediastinum and around the central airways on thin section CT scans.

Breathlessness was seen in $35 \%$ and fever was seen in $33.3 \%$.

The triad of cough, fever and haemoptysis is a reliable indicator of bronchiectasis. 


\section{UNILATERAL RIGHT LOBAR INVOLVEMENT}

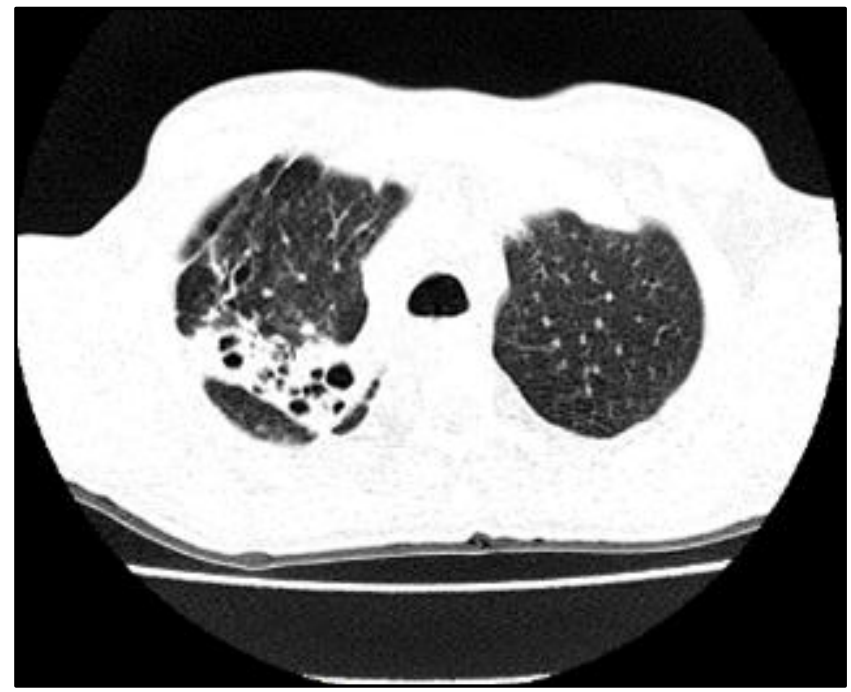

Right Upper Lobe

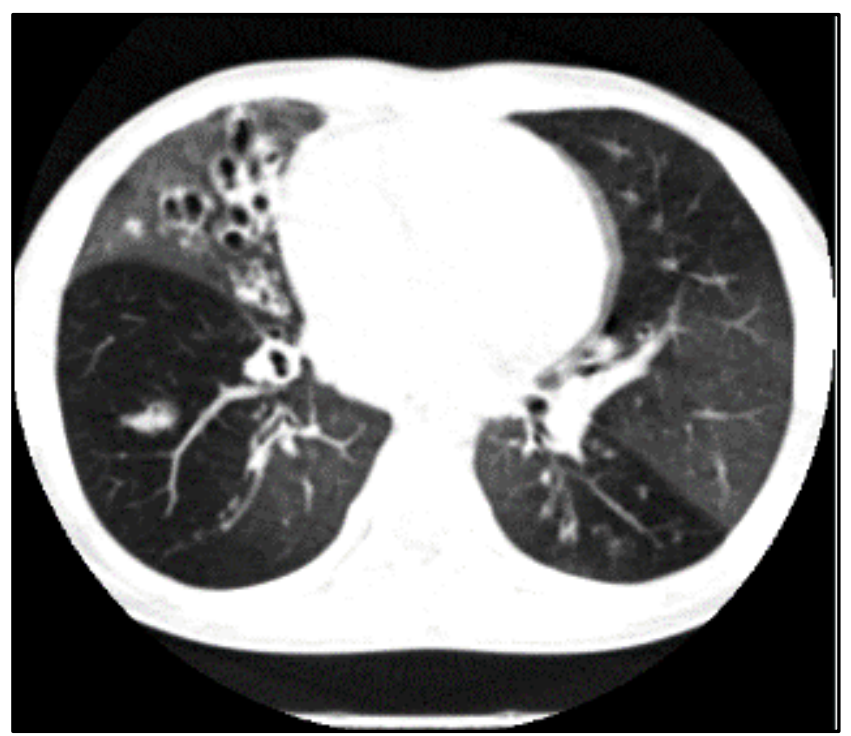

Right Middle Lobe

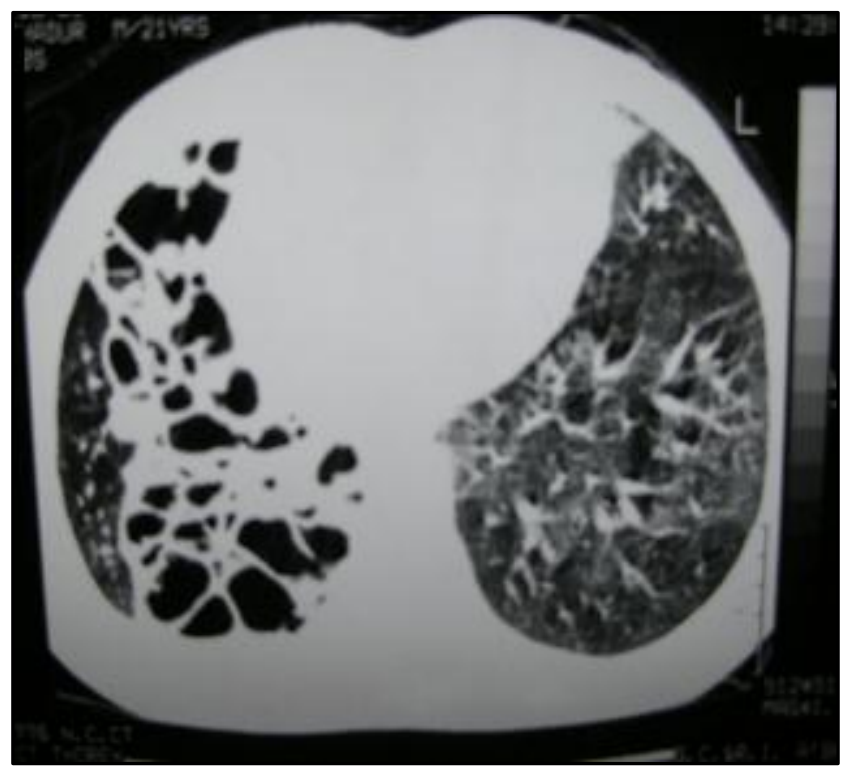

Right Lower Lobe

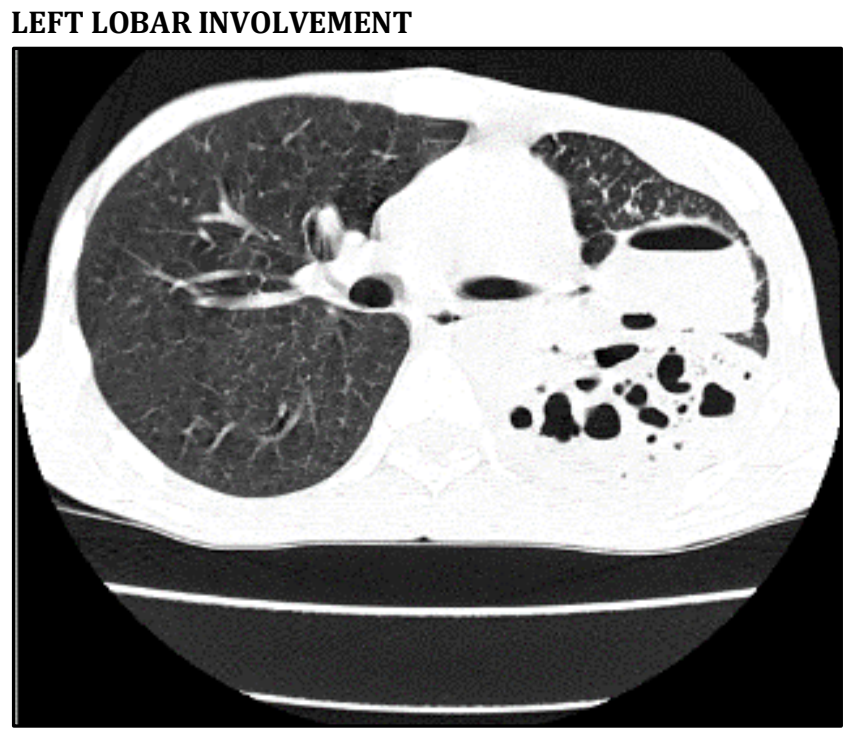

Left Upper Lobe

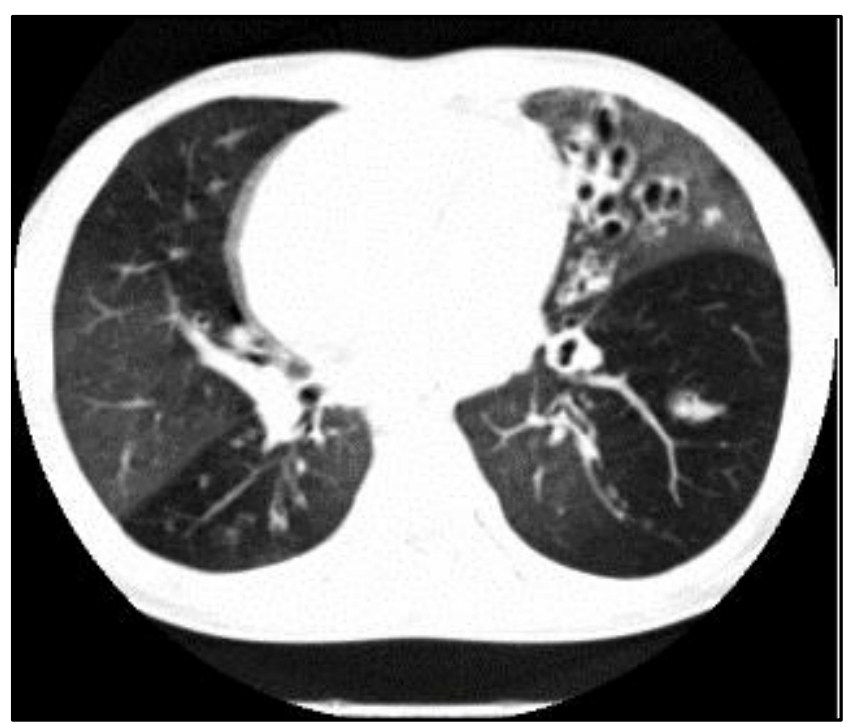

Lingula

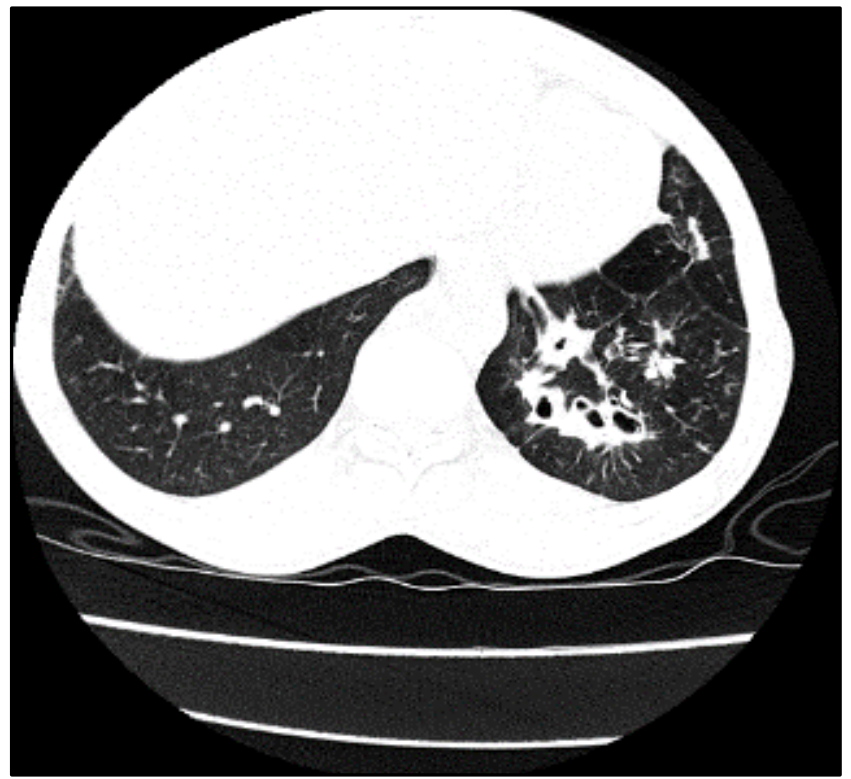

Left Lower Lobe 


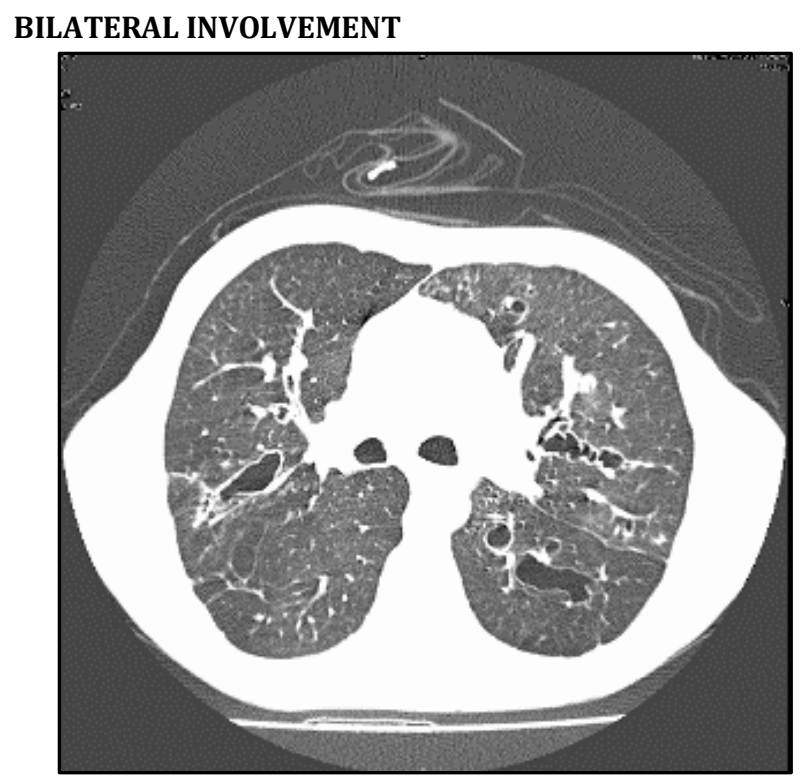

Upper Lobe

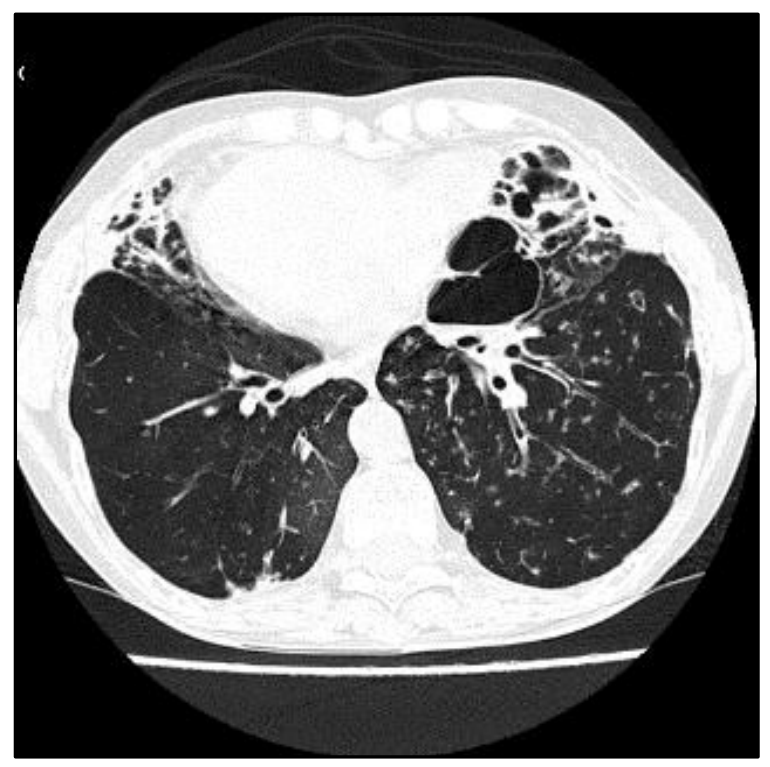

Middle Lobe/Lingula

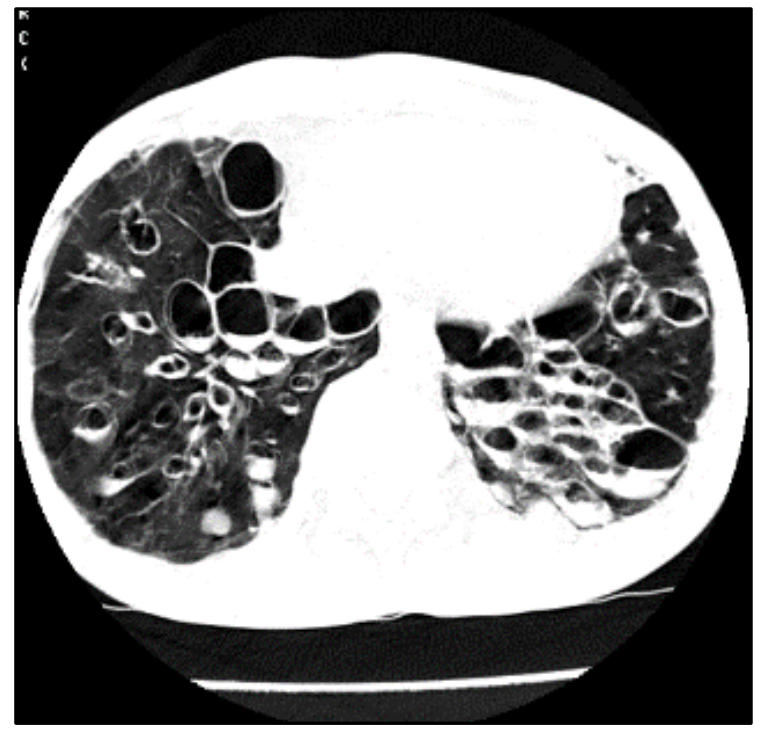

Lower Lobe

\section{HRCT FINDINGS}

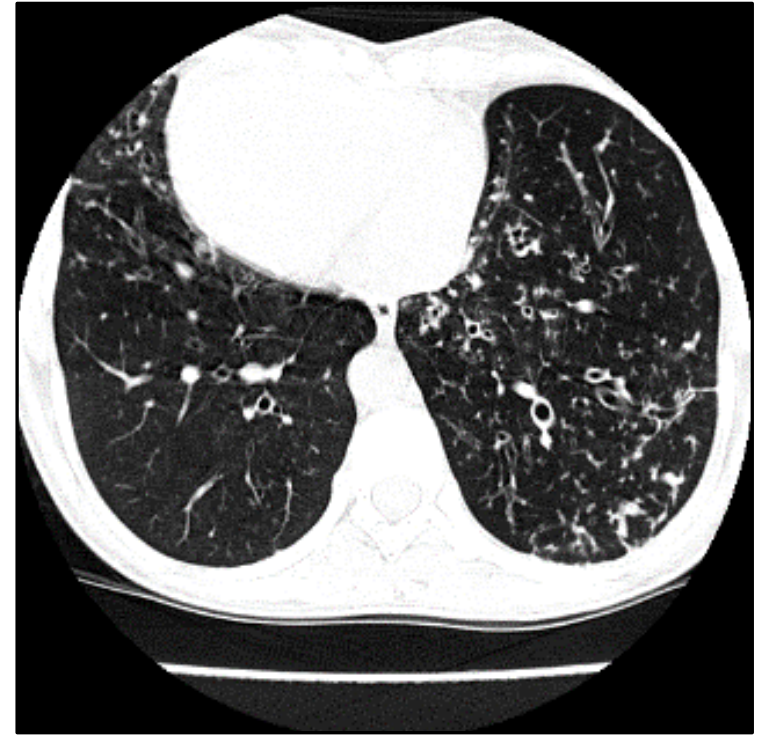

Bronchial: Pulmonary arterial ratio $>1.5$ (Signet Ring Appearance)
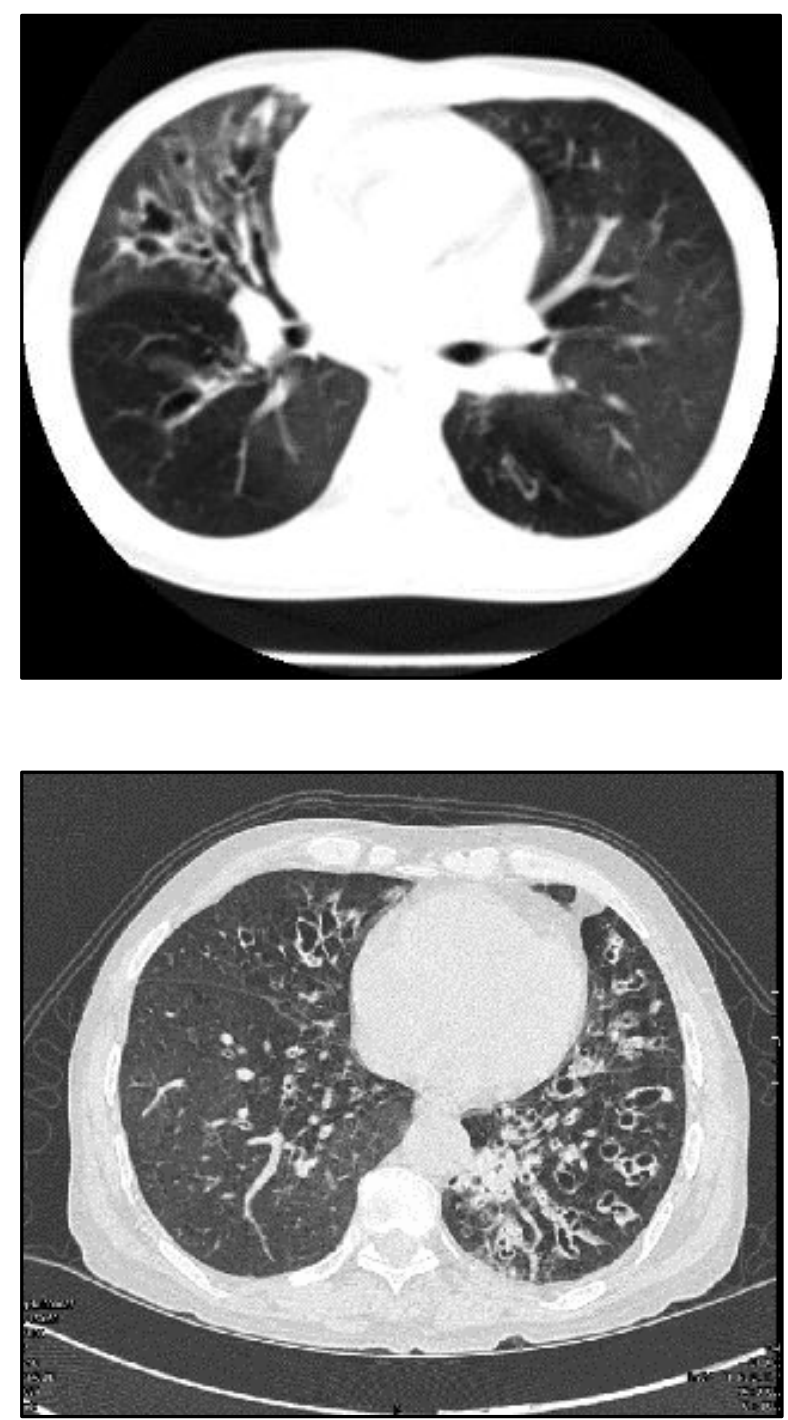

Lack of tapering of bronchi, peripherally visualised bronchi. 


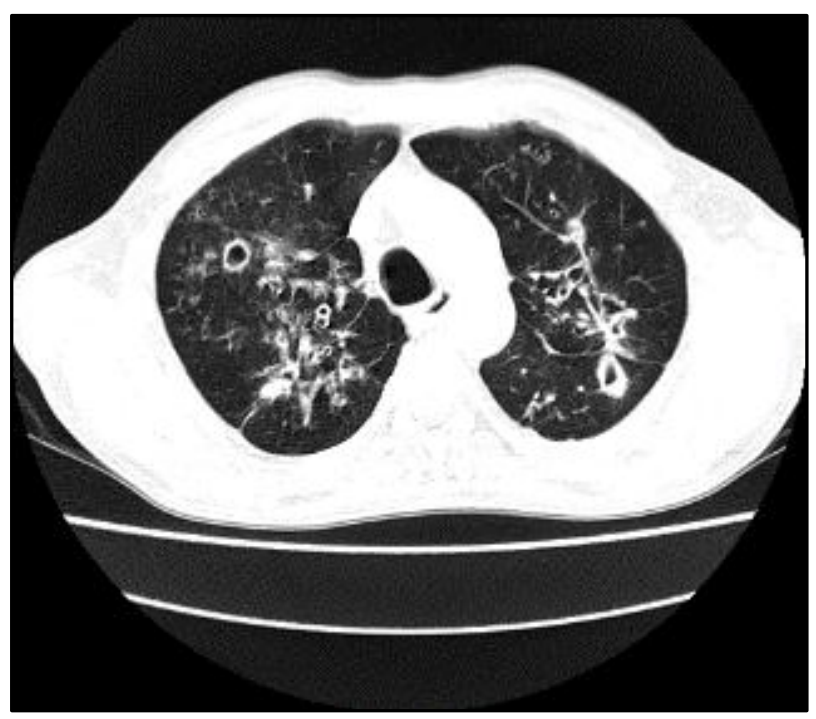

Possible Aetiological Factors: Utility of HRCT in Different Causes of Bronchiectasis

In our study by co-relating HRCT findings and clinical history, an accurate diagnosis was made in 46 cases, i.e. $76.6 \%$ which was confirmed by laboratory investigations.

In our study 23 patients (46\%) had pulmonary tuberculosis, in 8 patients (16\%) no cause was found, 16 patients $(32 \%)$ had history of recurrent childhood infections, 2 patients (4\%) had bronchial asthma and COPD and 1 patient (2\%) had allergic bronchopulmonary aspergillosis.

Although, the underlying cause of bronchiectasis is identified in $60 \%-80 \%$ of cases, HRCT findings in a number of disease entities have been described. The reliability of HRCT for distinguishing between these is still debated.

Lee et al (1995) in a study of HRCT scans in 108 patients, who had bronchiectasis from a variety of causes, found that a correct first choice diagnosis was made by 3 experienced observers in only $45 \%$ of cases. Further interobserver agreement was poor, leading these observers to conclude that HRCT was of little value in diagnosing specific aetiologies of

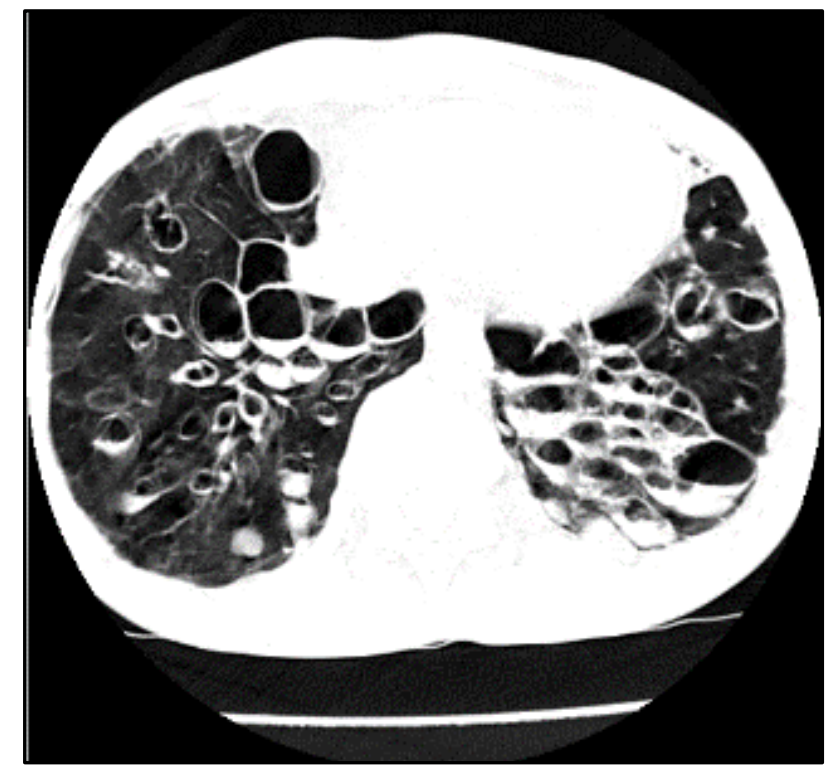
bronchiectasis. It should be emphasised that HRCT scans were interpreted in absence of clinical data. ${ }^{3}$

Cartier et al (1999) reported slightly better results in a retrospective study of 82 patients who head bronchiectasis with documented aetiologies. These authors noted that a correct diagnosis was reached by 2 independent observers in $61 \%$ of cases of cystic fibrosis, $67 \%$ of cases with tuberculosis and $56 \%$ of cases with allergic bronchopulmonary aspergillosis. Bilateral upper lobe distribution was most commonly seen in patients who had cystic fibrosis and allergic bronchopulmonary aspergillosis, whereas unilateral upper lobe distribution was most common in patients who had tuberculosis and a lower lobe distribution was most often seen in patients after recurrent childhood viral infections. ${ }^{4}$

In our study, 23 patients (46\%) had pulmonary tuberculosis. This is a significant number leading to the conclusion that bronchiectasis is an important and common sequel to tuberculosis. The parenchymal fibrosis and retraction that occur in tuberculosis result in bronchial enlargement. Another factor contributing to bronchiectasis in patients of tuberculosis is the infection of bronchial wall and endobronchial occlusion causing distal bronchiectasis. In study by Palwaywichai A (2002), the most common identifiable aetiology was tuberculosis. The study of 168 patients by Reiff et al (1995) had a large proportion of patients having post-tuberculosis bronchiectasis.

Our study co-related well with the findings of Cartier et al. Upper lobe bronchiectasis was seen in patients suffering from allergic bronchopulmonary aspergillosis. Predominantly lower lobe distribution, especially posterior basal segment of left lower lobe was affected in patients with history of recurrent childhood infections. Bronchiectasis as a result of tuberculosis was seen in upper lobes as well as in right middle lobe and lingual in our study.

Recurrent childhood infections as a cause of bronchiectasis could be obtained in 16 patients (32\%) in our study. In a review of 123 patients done by Nicotra (1995) who had documented bronchiectasis, an antecedent potentially causative event, usually pneumonia could be identified in $70 \%$ of cases.

In our study, in patients (16\%) there was no identifiable cause of bronchiectasis. In study by Frey et al (1997), 25\% of cases had no identifiable cause of bronchiectasis. In study by 
Reiff et al (1995), the cause of bronchiectasis could not be ascertained in $63 \%$ of cases.

In our study, 2 out of 60 patients (3.23\%) had bronchial asthma. Hansell et al (1994) showed an association between bronchial asthma and bronchiectasis. In study by Grenier et al (1996), bronchiectasis in asthmatic patients was found in $28.5 \%$ of patients. Bronchial asthma leads to small airway involvement consistent with obliterative bronchiolitis, which in turn leads to bronchiectasis.

In our study, allergic bronchopulmonary aspergillosis was identified as the cause of bronchiectasis in 1 patient (1.6\%). In a retrospective study of 82 patients of bronchiectasis, Cartier et al (1999) found allergic bronchopulmonary aspergillosis in 5 cases (6.9\%). Central bronchiectasis in association with bronchial occlusion due to mucous plugging, air fluid levels in dilated cystic airways and bronchial wall thickening are usually seen in bronchiectasis due to allergic bronchopulmonary aspergillosis. of particular interest is the finding of high attenuation mucoid impaction in the dilated bronchi. First described in association with chronic fungal sinusitis, high density mucous presumably represents the presence of calcium ions, metallic ions or both within viscous mucous. The prevalence of this finding has been noted to be as high as $28 \%$ in one series and when present should be considered characteristic.

\section{Morphological Forms of Bronchiectasis}

We based on our classification of bronchiectasis based on Reid's classification, i.e. cystic, cylindrical and varicose bronchiectasis. A total of 24 patients (58\%) in our study had cystic bronchiectasis, while 5 patients $(10 \%)$ had cylindrical variety, 2 patients (4\%) had varicose bronchiectasis and 19 patients (38\%) had mixed variety. There have been varied reports regarding the preponderance of cystic and cylindrical bronchiectasis; however, in majority of the studies the least number of patients had varicose bronchiectasis. This was a finding in our study also. ${ }^{5}$

\section{MORPHOLOGICAL FORMS OF BRONCHIECTASIS}

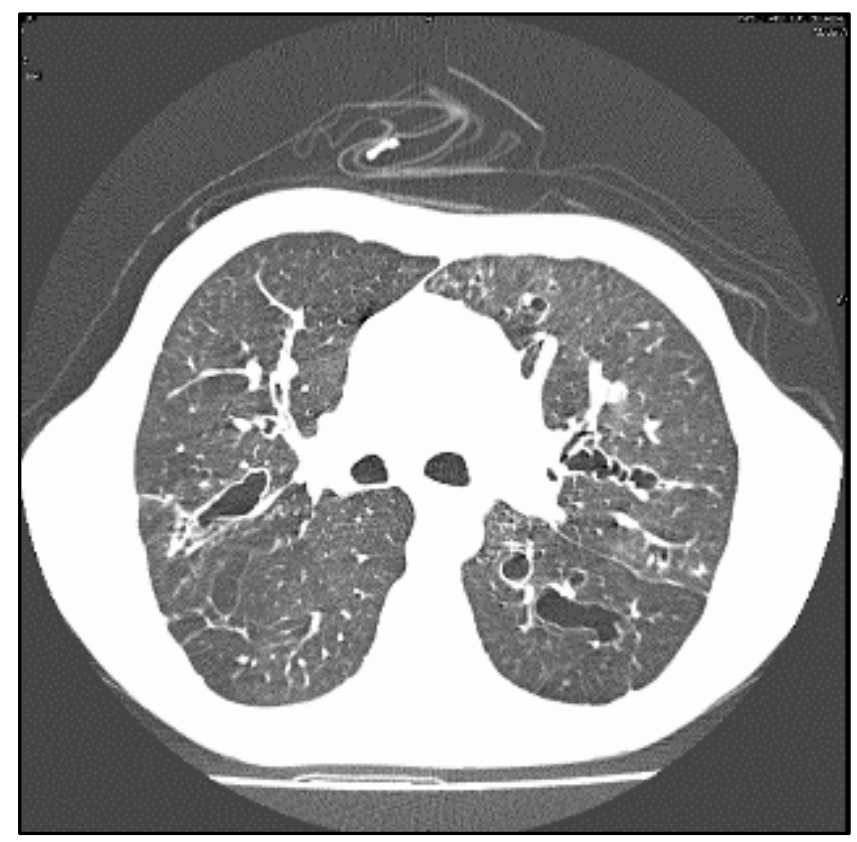

Cylindrical Bronchiectasis
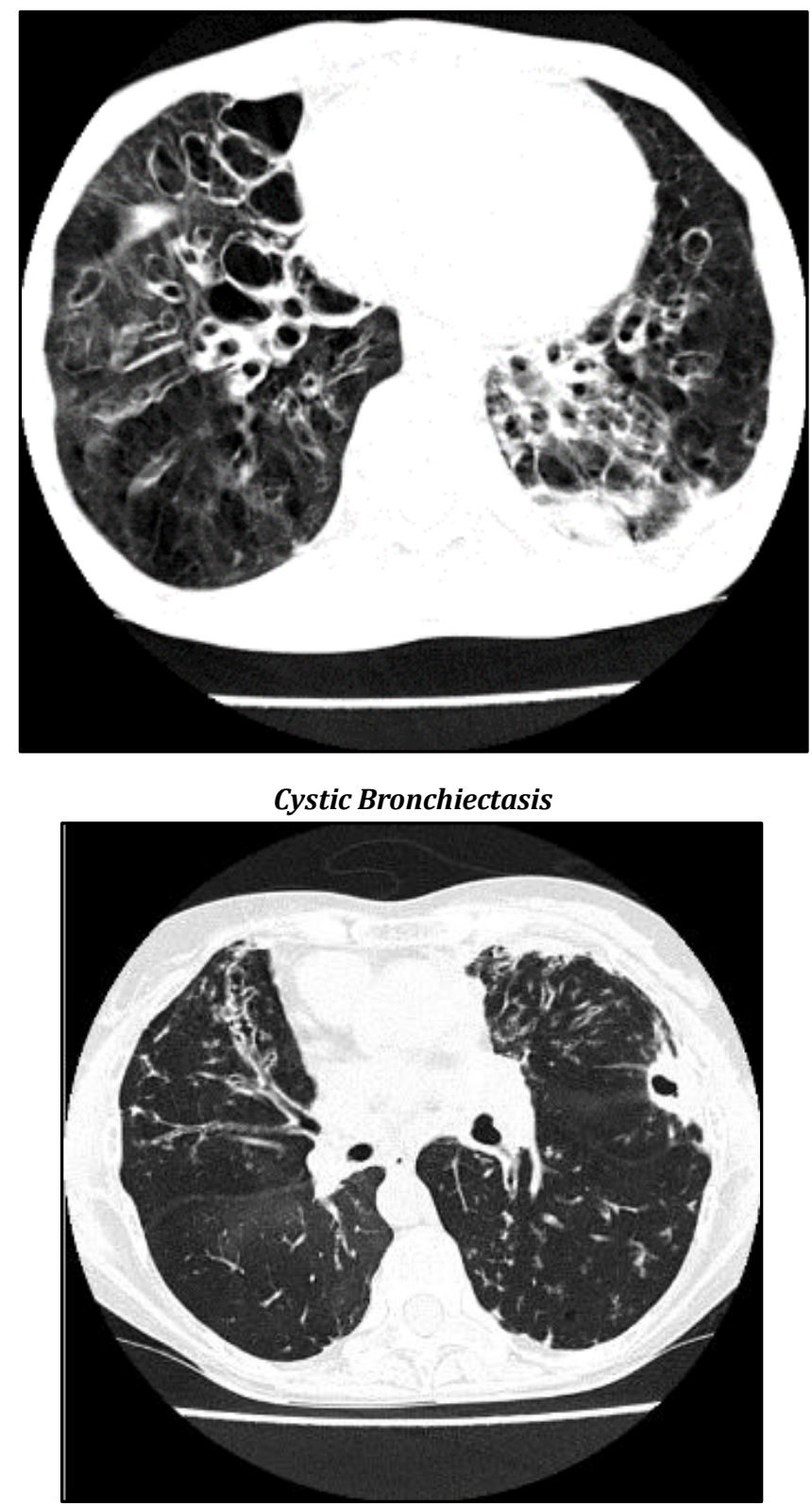

Varicose Bronchiectasis

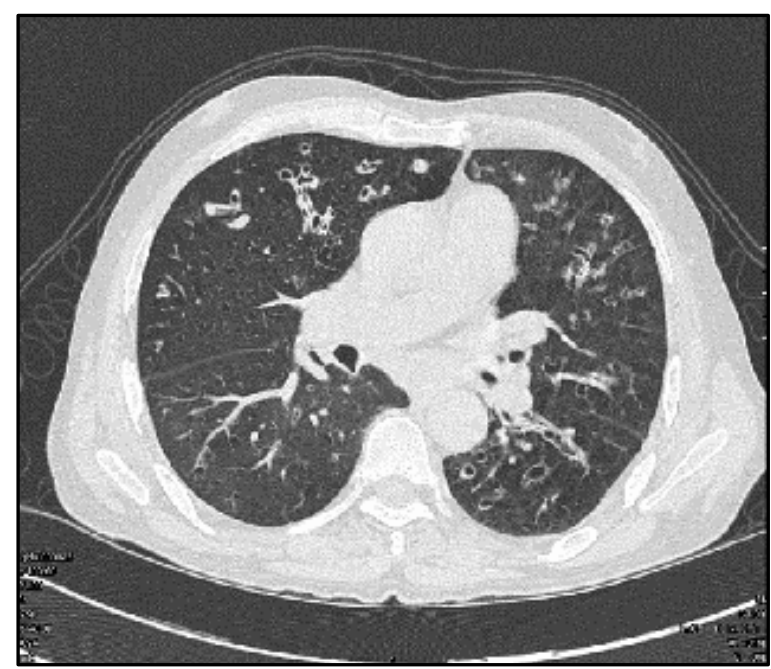

Mixed Bronchiectasis

\section{Localisation of Bronchiectasis}

With the advent of HRCT, accurate lobar and segmental localisation is possible. A total of 169 lobes were found to be 
bronchiectatic in our study. Out of these, majority were the lower lobes 69 (48.3\%). The left lower lobe was the most commonly affected lobe (24.2\%). This was consistent with the studies conducted by B. Ban (1995) (65\%), Clark (75\%) and OP Malhotra (70\%), in which the posterior basal segment of left lower lobe was the most commonly affected segment.

There was unilateral involvement in 15 patients (33.3\%) and bilateral involvement in 35 patients (66.6\%). In the study conducted by Clark et al there was equal number of unilateral and bilateral involvement, while in the study conducted by OP Malhotra $70 \%$ of patients had unilateral involvement.

\section{HRCT Findings in Bronchiectasis}

\section{Bronchial: Pulmonary Artery Ratio}

Bronchiectasis is defined by the presence of bronchial dilatation, hence recognition of increased bronchial diameter is the key to CT diagnosis of this abnormality. Various sophisticated methods for measuring airway dimensions have been proposed. Eg: Digital image analysis programmes. These approaches may allow precise quantitative assessment of airways and may prove particularly valuable in physiologic studies. Subjective visual criteria for establishing the presence of bronchial dilatation are most often used in the interpretation of scans. For the purpose of interpretation of HRCT, bronchial dilatation may be diagnosed using a comparison of bronchial size to that of adjacent pulmonary artery branches.

In most cases, bronchiectasis is considered to be present when the internal diameter of a bronchus is greater than the diameter of adjacent pulmonary branch, i.e. the bronchial : pulmonary artery ratio $>1$. The accuracy of this finding in diagnosing bronchiectasis has been validated in a number of studies comparing CT to bronchography in patients who have bronchiectasis. In patients who have bronchiectasis, the bronchial diameter is often much larger than the pulmonary artery diameter (i.e. greater than 1.5 times the artery diameter), a finding that not only reflects the presence of bronchial dilatation but also demonstrates some reduction in pulmonary artery size as a consequence of decreased lung perfusion in affected areas. The definition of an abnormal bronchial : artery ratio varies widely among reported series.

Despite variability in normal bronchial size and methods by which bronchial diameter has been assessed, several studies have shown that measurements of bronchial diameter may be reliably made from HRCT. As emphasised by Diederich et al, visual inspection remains the mainstay for assessing bronchial diameter as obtaining objective measurement (with or without use of calipers) is time consuming and often clinically impractical. ${ }^{2}$

In our study was the bronchial : pulmonary artery ratio > 1.5 , was seen in $62 \%$ of patients. In study by Kang et al (1995), increased bronchial : pulmonary artery ratio was seen in $60 \%$ of patients.

\section{Lack of Bronchial Tapering}

Lack of bronchial tapering has come to be recognised as an important finding in the diagnosis and subtle cylindrical bronchiectasis in particular. It has been suggested that for this finding to be present the diameter of an airway should remain unchanged for at least $2 \mathrm{~cm}$ distal to branch point. In our study lack of bronchial tapering was noted in 38 patients (76\%). Kang et al (1995) found lack of tapering of bronchial lumina in $79 \%$. It should be emphasised that the accurate detection of this finding is difficult in the absence of contiguous HRCT sections, especially for vertically or obliquely oriented bronchi. The value of this sign is doubtful when HRCT scans are obtained at spaced intervals in a non-contiguous fashion.

\section{Visualisation of Peripheral Airways}

The smallest airways normally visible using HRCT techniques have a diameter of approximately $2 \mathrm{~mm}$ and a wall thickness of $0.2-0.3 \mathrm{~mm}$. In normal subjects airways in the peripheral 2 $\mathrm{cm}$ of lung are uncommonly seen, because their walls are too thin. Peribronchial fibrosis and bronchial wall thickening in patients who have bronchiectasis in combination with bronchial dilatation allow visualisation of small airways in lung periphery.

Peripherally visualised bronchi were noted in 40 (80\%) of our patients in study conducted by Kang et al (1995); $45 \%$ of patients had peripherally visualised bronchi. Kim et al (1997) found that visible bronchi within $1 \mathrm{~cm}$ of pleural surface or bronchi touching the mediastinal pleural surfaces were visible in $81 \%$ and $53 \%$ respectively of HRCT interpretations in patients who had clinical or pathological evidence of cylindrical bronchiectasis.

\section{Bronchial Wall Thickening}

Although, bronchial wall thickening is a non-specific finding, it is usually present in patients who have bronchiectasis.

Airways divide by asymmetric, dichotomous branching with approximately 23 generations of branches from the trachea to the alveoli. Anatomically, $2^{\text {nd }}-4^{\text {th }}$ generation segmental airways have a mean diameter between $5-8 \mathrm{~mm}$ corresponding to a wall thickness of approximately $1.5 \mathrm{~mm}$; $6^{\text {th }}-8^{\text {th }}$ generation airways have a mean diameter measuring between $1.5-3 \mathrm{~mm}$ with walls of approximately $0.3 \mathrm{~mm}, 11^{\text {th }}$ - $13^{\text {th }}$ generation airways have diameters measuring $0.7-1$ mm with walls of $0.1-0.15 \mathrm{~mm}$.

The relationship between wall thickness and bronchial diameter has been assessed by Kim et al (1997) using the Bronchial Lumen Ratio (BLR). At the subsegmental level, the BLR defined as the inner diameter of bronchus divided by outer diameter was measured at subsegmental level on a display console. Considerable variation in the BLR was found averaging $0.66 /+0.06$ with a range of $0.51-0.86 \mathrm{~mm}$. It has also been suggested that bronchial wall thickening may be diagnosed if the airway wall is at least 0.5 times the width of an adjacent vertically oriented pulmonary artery. ${ }^{6}$

Identification of thickened bronchial walls for the purposes of interpretation of clinical scans remains largely subjective. Because bronchiectasis and bronchial wall thickening are often multifocal rather than diffuse and uniform, a comparison of one lung region to another can be helpful in making this diagnosis.

In our study, bronchial wall thickening was noted in $88 \%$ of patients.

\section{Measurement of Bronchial Diameter}

The extent of bronchiectasis was quantified by first assigning a score to each of the six lobes according to the percentage (i.e. grade) of lobar involvement, which was derived with the following scale: Grade 0, none; Grade 1, mild (<25\%); Grade 2, moderate (25\%-50\%); and Grade 3, severe $(>50 \%$ involvement of each lobe). All individual lobar scores were summed to calculate the overall score for the extent of bronchiectasis. The thickness of the bronchial wall relative to the external diameter of dilated bronchi (EDB) perpendicular 
to the transverse plane was evaluated in each lobe. This score was determined with the following scale: Grade 0, normal thickness; Grade 1, thickness greater than $20 \%$ and less than $50 \%$ EDB; Grade 2, thickness greater than 50\% EDB; and Grade 3, complete obliteration of the bronchial lumen. If there was a range of bronchial wall thickening noted in each lobe assessed, a mean score was calculated per lobe whereby the number of scores assigned was the denominator for the sum of all scores calculated. The sum of individual lobar bronchial wall thickening scores was the overall score for each patient.

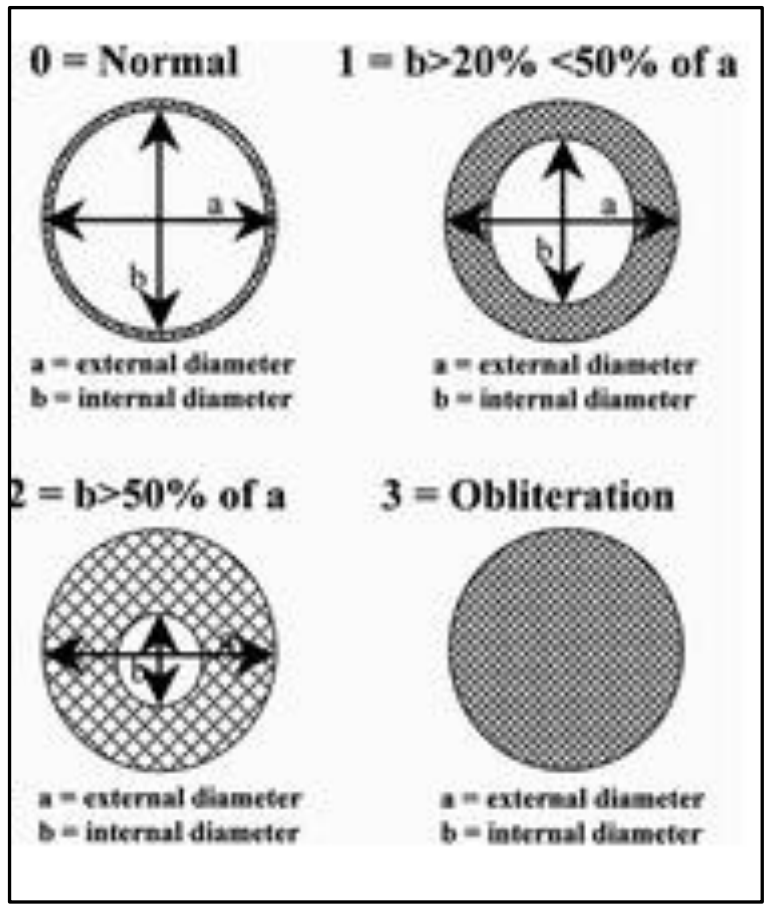

\section{HRCT vs. Chest Radiograph}

HRCT has revolutionised the detection and localisation of bronchiectasis, because of its axial sections and superior resolution compared to chest radiographs. In chest radiographs, $70 \%$ of lung tissue is obscured by the bony cage. However, this problem is not seen in HRCT. The bronchus can be followed from origin to periphery with the help of contiguous sections. Chest radiograph is neither sensitive nor specific for the diagnosis of bronchiectasis. The detection rate of HRCT in comparison to chest radiographs is better. Out of the 60 cases studied, Chest radiographs detected bronchiectatic changes in 55 cases; 5 cases were interpreted as normal by chest radiograph which were abnormal on HRCT. Even among radiographs which showed unilateral bronchiectasis, HRCT revealed the disease to be bilateral. Exact localisation of disease to a particular anatomical segment of lungs was possible with HRCT, which was not possible with chest radiographs. In a study by Vincent Mysliwiee et al (1999) chest radiographs were abnormal in only $87 \%$ of cases. In a study conducted by Palwaywichai et al (2002), abnormal chest radiographs were seen in $9 \%$ of cases. In study of Boehm T et al (2003), chest radiograph was found to have a sensitivity of only $37 \%$ for detection of bronchiectasis.

In a study of 84 patients done by Van Der Bruggen (1996), 47 patients had abnormal radiographs. Out of these 36 had bronchiectasis on HRCT and the other 11 patients had normal scans. The sensitivity of radiograph to detect bronchiectasis was $87.8 \%$ and the specificity was $74.4 \%$. They found a significant linear relationship between the severity of bronchiectasis at HRCT and abnormalities as seen on chest radiograph. In our study, the sensitivity of chest radiographs in detecting bronchiectasis was $94 \%$. This high sensitivity rate as compared to other studies could be due to the fact that most of our patients had severe bronchiectasis, which could be easily detected on radiographs. ${ }^{7}$

In the study by Issac Hassan et al (2003), HRCT had a sensitivity of $96 \%$ and specificity of $93 \%$. In our study, HRCT had $100 \%$ sensitivity and specificity. In study by Boehm T et al (2003), HRCT has been shown to have a sensitivity of $96-98 \%$ and a specificity of $93-99 \%$ in detection of bronchiectasis. Using HRCT, Grenier et al (1996) found a sensitivity of $96 \%$ and a specificity of $93 \%$ as compared to bronchography for diagnosing bronchiectasis. Young et al also assessed the reliability of HRCT in assessment of bronchiectasis. Their study showed a sensitivity of $98 \%$ and a sensitivity of $99 \%$.

The first study in evaluating bronchiectasis by CT was done by Naidich DP et al in 1982.

\section{CONCLUSIONS}

- In a given clinical setting suggestive of bronchiectasis, HRCT serves as the best modality in confirming the diagnosis of bronchiectasis. HRCT is definitely superior to chest radiographs in the detection of bronchiectasis.

- Regional distribution of bronchiectasis can be well visualised by HRCT. Left lower lobe, especially posterior basal segment is most commonly involved.

- In this study, incidence is most common in the age group of 21-30 years and is more common in males than females.

- Bilateral involvement is more common than unilateral involvement.

- Peripherally visualised bronchi was the most common finding in our study followed by lack of tapering.

- Cystic bronchiectasis is the most common form of bronchiectasis followed by mixed variety. Varicose bronchiectasis is the least common form.

- HRCT is useful in identifying the aetiology of bronchiectasis in most patients. Tuberculosis is the most common aetiological factor of bronchiectasis.

- Other co-existent lung pathologies can also be diagnosed by HRCT.

\section{REFERENCES}

1. Currie DC, Cooke JC, Morgan AD, et al. Interpretation of bronchograms and chest radiographs in patients with chronic sputum production. Thorax 1987;42(4):278-84.

2. Frey HR, Russi EW. Bronchiectasis-current aspects of an old disease. Schweiz Med Wochenschr 1997;127(6):219 30.

3. Lee PH, Carr DH, Rubens MB, et al. Accuracy of CT in predicting the cause of bronchiectasis. Clin Radiol 1995;50(12):839-41.

4. Cartier Y, Kavanagh PV, Johkoh T, et al. Accuracy of high resolution ct in differentiation of specific diseases. AJR Am J Roentgenol 1999;173(1):47-52.

5. Reid LM. Reduction in bronchial subdivision in bronchiectasis. Thorax 1950;5(3):233-47.

6. Kim JS, Im JG, Kim IO, et al. Normal bronchial and pulmonary arterial diameters measured by thin section ct. J Comput Assist Tomogr 1995;19(3):365-9.

7. Van Der Bruggen-Bogaarts BA, van Der Bruggen HM, van Waes PM, et al. Screening for bronchiectasis: a comparative study between chest radiography and highresolution CT. Chest 1996;109(3):608-11. 